\title{
Patrón de Asentamiento de los Grupos que Habitaron el Valle Puebla- -Tlaxcala, México durante el Periodo Epiclasico (650-950 AD). Fase de recorrido de superficie
}

\author{
Jesus Carlos Lazcano Arce (*) \\ Marianne Sallum (**)
}

\begin{abstract}
LAZCANO ARCE, J.C., SALLUM, M. Patrón de Asentamiento de los Grupos que Habitaron el Valle Puebla-Tlaxcala, México durante el Periodo Epiclasico (650-950 AD). Fase de recorrido de superficie. R. Museu Arq. Etn., 25: 45-60, 2015
\end{abstract}

Resumen: Este trabajo tiene como objetivo de conocer, analizar y entender el patrón de asentamiento de los sitios arqueológicos del periodo Epiclásico (650-950 A.D.), localizados en el valle de Puebla-Tlaxcala (México). Se tiene la intención de evaluar primero, las condiciones de conservación en las que se encuentran y segundo, determinar cuales de ellos, según las características arquitectónicas que presentan y el área natural en la que se localizan, fueron dependientes o tributarios del sitio rector de Xochitecatl-Cacaxtla. Para lograr esto se utilizaron diversas fuentes de información: una inicial, sustentada en los trabajos previos de recorridos de superficie y excavaciones realizadas en diversos sitios arqueológicos y una segunda, sobre la base de los nuevos datos recogidos con el actual estudio de recorrido de superficie. El estudio logro establecer un área extensa de similitud cultural entre Xochitecatl-Cacaxtla y por lo menos veinte sitios hacia la zona centro-sur del valle cuyo límite es el área nuclear del sitio principal de Cholula al sur del propio valle Puebla-Tlaxcala.

Palabras-clave: Valle de Puebla-Tlaxcala, Periodo Epiclássico, Mesoamérica

\section{Introducción}

E 1 presente artículo da cuenta de los trabajos que se realizaron en la primera temporada de trabajo de campo del proyecto de investigación intitulado "Estudio regional de los grupos que habitaron el valle Puebla-Tlaxcala durante el periodo Epiclásico (650-950 d.C.), investigación que se origina en el Instituto de Investigaciones Antropológicas de la Universidad Nacional Autónoma de México. El estudio realizado en su primera fase consistió en realizar una relocalización de los sitios arqueológicos

${ }^{*}$ ) UNAM - Universidad Nacional Autónoma de México. <clazcano@humanidades.unam.mx>

${ }^{(* *)}$ Museu de Arqueologia e Etnologia, Universidade de São Paulo.<marianne.sallum@gmail.com> reportados en la zona conocida como valle Puebla-Tlaxcala (estado de Tlaxcala y Puebla, México) y, con ello, un diagnóstico de las condiciones de conservación en las que se encuentran. Asimismo y con el material cerámico diagnóstico en ellos localizado, determinar su temporalidad relativa. La intensión final de este estudio responde a la elección de varios sitios arqueológicos para su inmediata exploración y con ellos conocer el desarrollo de las sociedades que habitaron esta región dando prioridad a la identificación de sus actividades productivas principales, explotación de recursos, identidad étnica y si existió entre ellos diferentes niveles de jerarquía.

Así, bajo una perspectiva temporal definida, como lo es el Epiclásico (650-950 AD) 


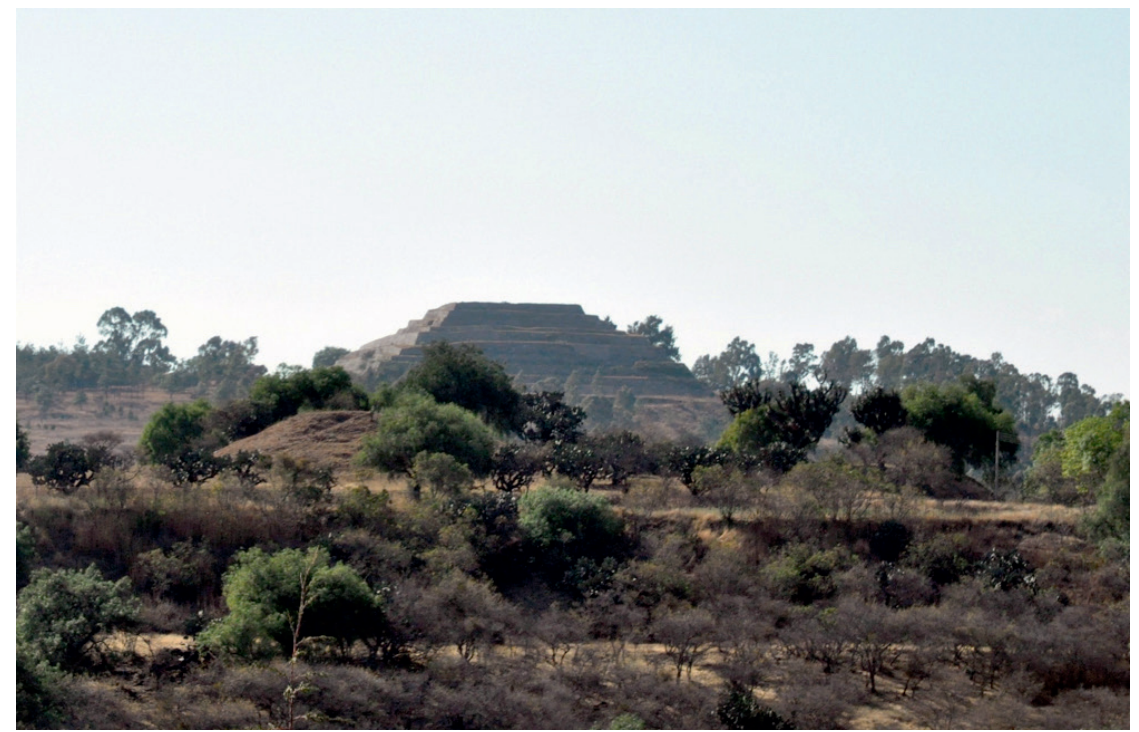

Fig. 1. Sitio arqueológico de Xochitecatl-Cacaxtla.

y desde un punto de vista regional, lograr un acercamiento a la realidad social del pasado y con ello intentar clarificar y en su caso corroborar las características establecidas para esta área cultural. De igual modo y con base en los estudios realizados en un sitio arqueológico de gran magnitud como lo es Xochitecatl-Cacaxtla (fig. 1), buscamos identificar si algunos de los sitios arqueológicos del valle Puebla-Tlaxcala fueron tributarios, dependieron o fueron fundados por el sitio rector de Xochitecatl-Cacaxtla y definir cuales aspectos sociales que se le han atribuido a la sociedad pretérita de este asentamiento se encuentran en otros sitios del valle Puebla-Tlaxcala.

De esta manera, utilizaremos tres tipos de información: la inicial, ya recabada mediante trabajos antecedentes, seguida por la que aporten los nuevos recorridos de superficie que aquí reportamos y finalmente la información que se obtenga con base en el estudio y excavaciones extensiva e intensiva de algunos sitios elegidos según sus condiciones y características.

Desde nuestra perspectiva, esta investigación intenta aportar conocimientos en temas específicos, de acuerdo con una temporalidad definida, en virtud de las características y desarrollo cultural de los habitantes prehispánicos del valle Puebla-Tlaxcala (véase fig. 2).
Antecedentes de estudio en la región del valle Puebla-Tlaxcala

Las investigaciones arqueológicas en el valle Puebla-Tlaxcala iniciaron hace más de tres décadas. La primera (1962) estuvo a cargo de la Fundación Alemana para la Investigación Científica (FAIC), institución que realizó estudios hidrológicos, geográficos, biológicos, económicos y antropológicos. Para el caso de las investigaciones antropológicas y, en especial, las arqueológicas, los alemanes llevaron a cabo varios recorridos de superficie entre la llanura del río Atoyac y Zahuapan, y excavaron los sitios de Totemihuacan y Xochitécatl. El interés fundamental de estos estudios fue conocer la amplitud y características de los asentamientos prehispánicos.

De los primeros estudios realizados por la Fundación Alemana se obtuvo un catálogo de sitios arqueológicos que Tschohl y Nickel publicaron en 1972. De forma paralela se iniciaron trabajos de excavación en sitios monumentales realizados por el doctor Spranz en Xochitécatl (1966-1970), Walter (1970-1971) y Aufdermauer (1970-1973) (García Cook, 1997:58).

En 1972 se generó el proyecto mancomunado mexicano-alemán Puebla-Tlaxcala, cuyo responsable principal fue el investigador Ángel García Cook. Los estudios no sólo se encami- 


\section{El valle Puebla-Tlaxcala}

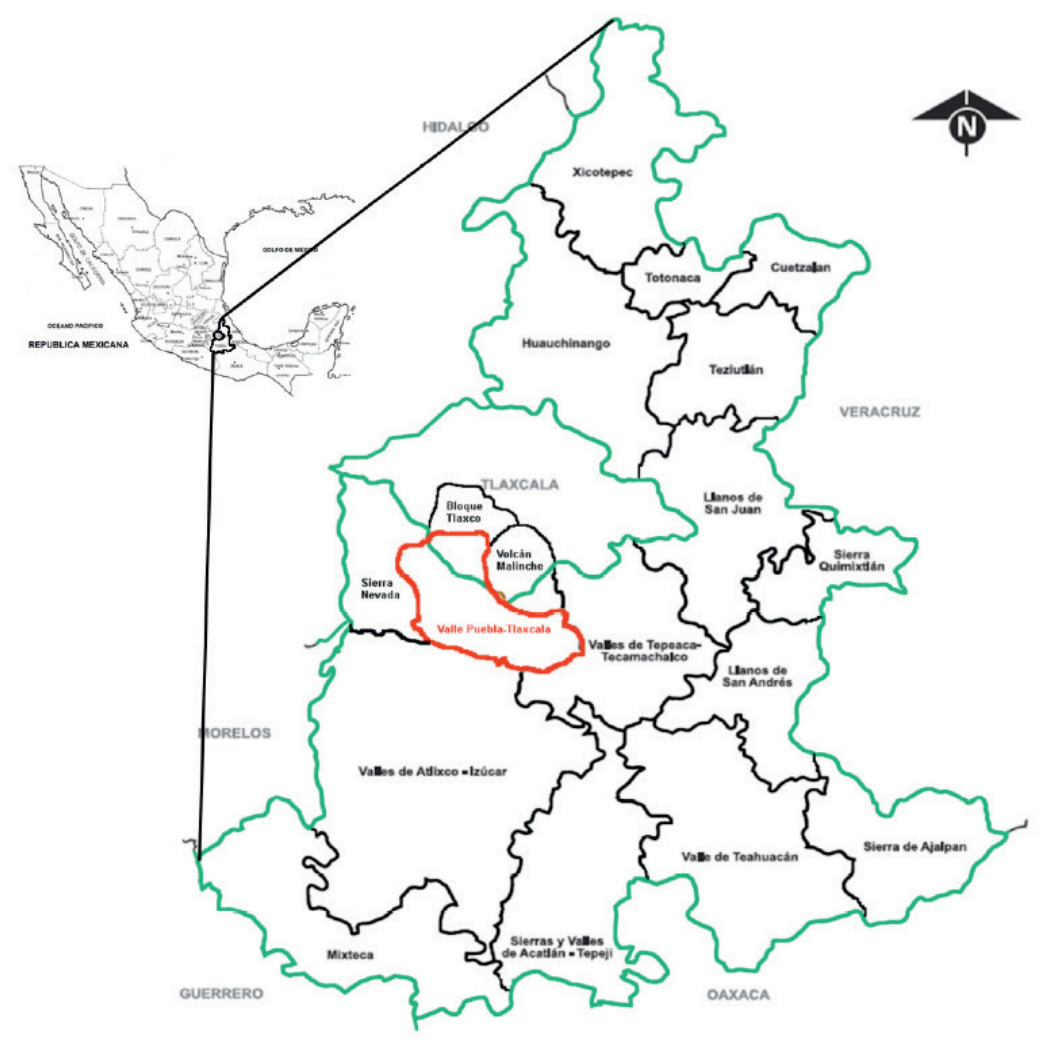

Fig. 2. Localización del valle Puebla-Tlaxcala.

naron a la localización de sitios arqueológicos, sino también a la excavación intensiva de varios de éstos, con el fin de conocer su temporalidad y sus características.

El entonces proyecto Puebla-Tlaxcala recorrió un área de $4.500 \mathrm{~km} 2$ dividida en tres subáreas. De igual manera, catalogó y estudió más de 750000 elementos culturales y localizó un poco más de 700 sitios arqueológicos (véase fig. 3).

Con la información recopilada y con las comparaciones efectuadas se propuso una secuencia de fases culturales para definir el desarrollo regional y observar los procesos que las impulsaron.

Cabe señalar que los estudios del profesor García Cook no cubrieron del todo el área geográfica del valle Puebla-Tlaxcala. Las fases cultural Texcalac que corresponde al periodo Epiclásico cubre parte de la zona norte del valle Puebla-Tlaxcala (García Cook 1997b:96) (véase fig.4).
Durante los trabajos del proyecto Puebla -Tlaxcala se tuvo la oportunidad de estudiar otras áreas fuera de lo que en principio se había considerado, por lo cual se conformaron los proyectos: Huejotzingo a cargo del Dr. Peter Schmidt y el proyecto Cuauhtinchan dirigido por Patricio Dávila y Diana Zaragoza. Por el mismo tiempo se creó el proyecto Cholula a cargo de Ignacio Marquina, cuyos avances de investigación se publican en 1970.

El proyecto Huejotzingo tuvo como objetivo realizar un recorrido de superficie para determinar el patrón de asentamiento de las diferentes épocas de ocupación y con ello establecer una secuencia cultural. Después se llevaron a cabo excavaciones arqueológicas en algunos sitios relacionados con la etapa posclásica tardía (1325. $1521 \mathrm{dC}$ ) y con el periodo Colonial. Esto con el fin de conocer arqueológicamente una entidad 


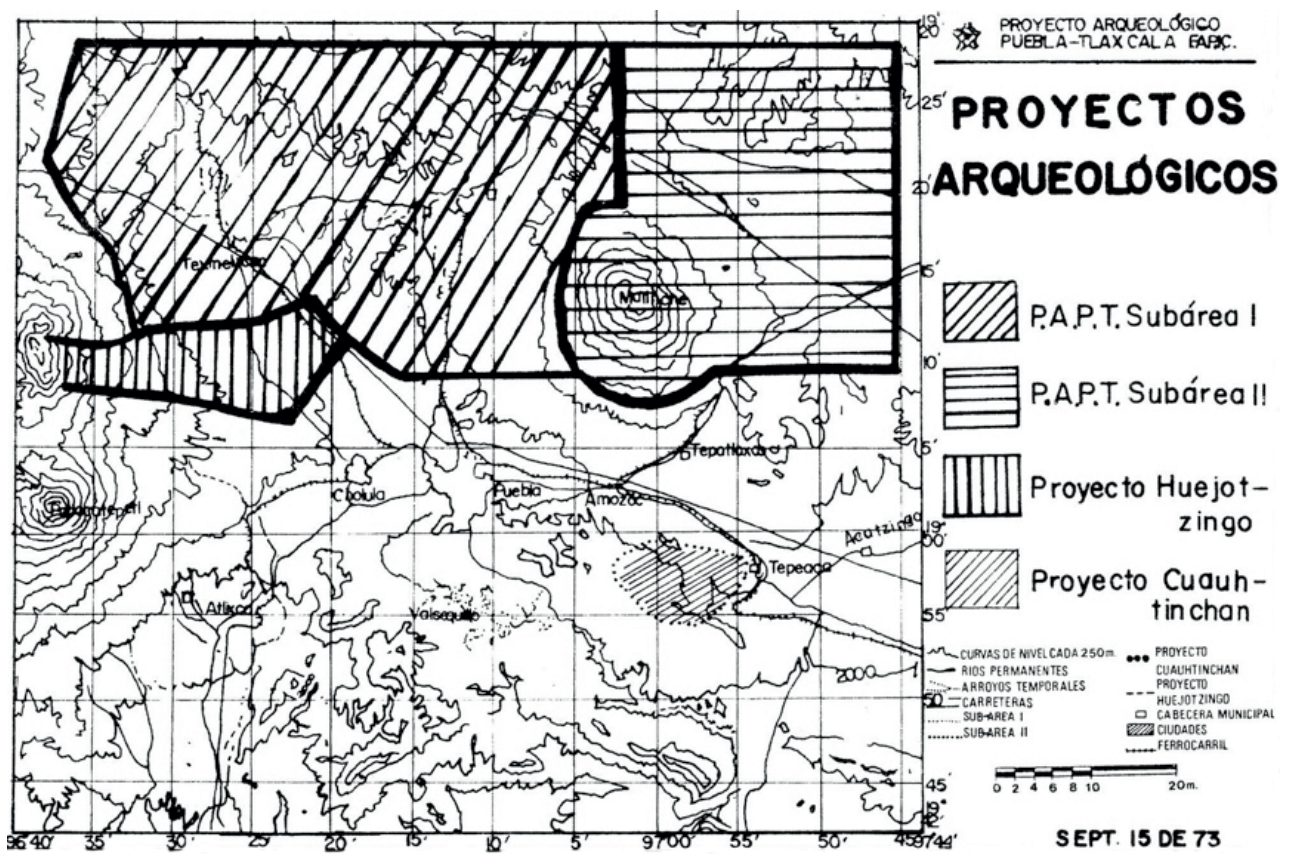

Fig. 3. Localización de las áreas de recorrido del proyecto Puebla-Tlaxcala. Tomado de García Cook:1997a, anexo cartográfico.

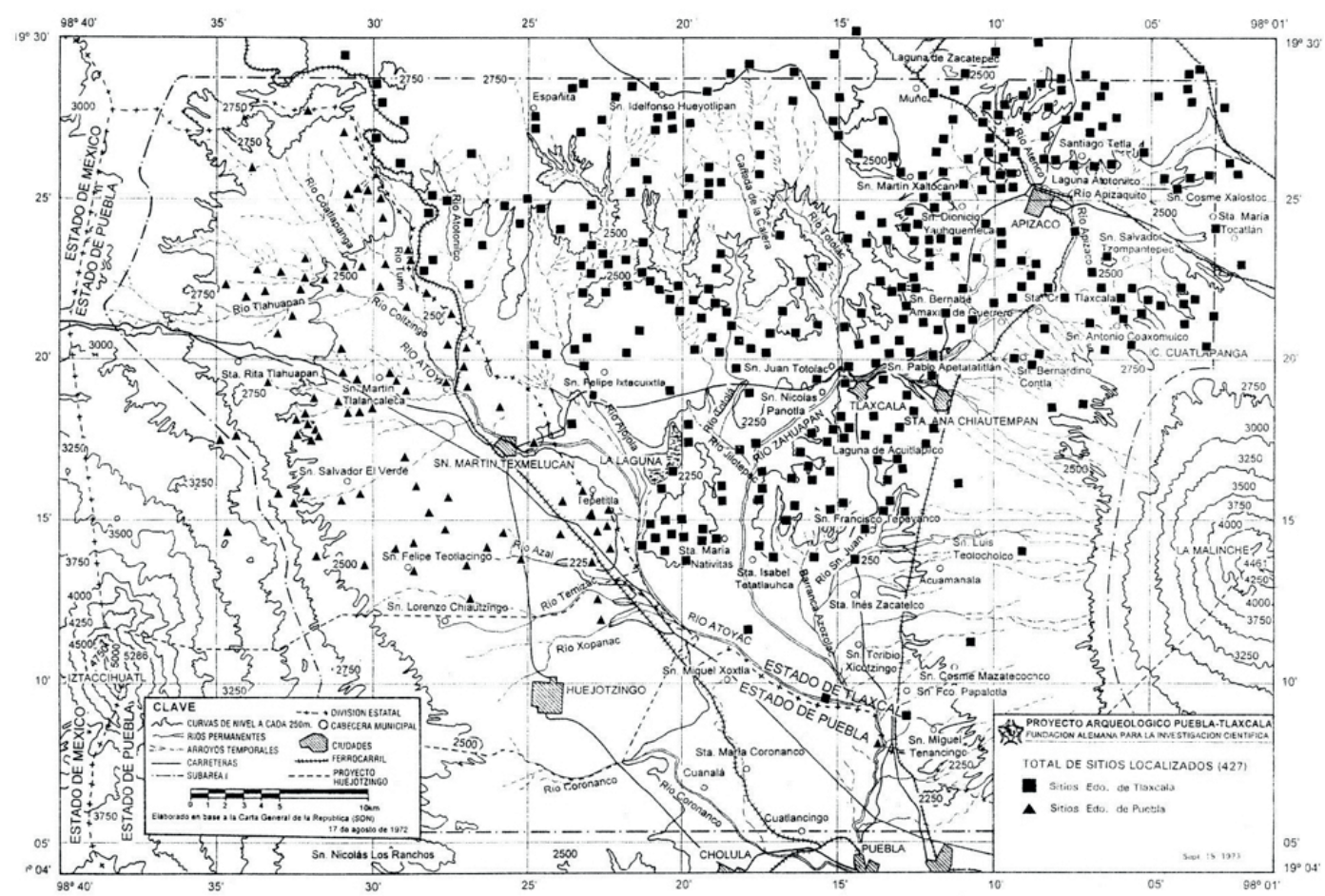

Fig. 4. Localización de los sitios de la fase Texcalac (650-850 A.D.) Tomado de García Cook:1997a, anexo cartográfico. 
independiente de los mexicas y que durante el virreinato, fue un enclave determinante para la evangelización. El resultado de dicho proyecto fue la propuesta de ocho fases culturales definidas por la combinación de ciertos tipos cerámicos. La fase Trinidad (600-1100 AD) es la que corresponde al momento que aquí nos interesa (Schmidt 1979:169-173).

El proyecto Cuauhtinchan realizó un reconocimiento arqueológico con el fin de constatar la presencia de sitios arqueológicos identificados en el mapa 2 de la serie de Mapas Coloniales de Cuauhtinchan. El proyecto posteriormente se amplía con la finalidad de comprender el desarrollo que tuvo su población. Para lograr lo anterior, se realizaron recorridos de superficie sistemáticos, así como sondeos estratigráficos. El resultado fue la localización de 61 sitios, de los cuales 10 corresponden a la temporalidad del Clásico tardío (Dávila Cabrera 1974:134-139).

El proyecto Cholula buscó realizar un estudio integral de la población prehispánica en la región de Cholula, Puebla y realizar excavaciones sistemáticas en la gran pirámide que allí se encuentra. El resultado fue la exploración de algunas áreas alrededor de la estructura piramidal (Marquina 1970). En la actualidad se han realizado nuevos trabajos de investigación en esta área por parte del personal de investigación de la Universidad de las Américas de Puebla (Plunket y Uruñuela 2005) (véase fig. 5).

En forma paralela a los trabajos de investigación de la Fundación Alemana y del proyecto Puebla-Tlaxcala, el Instituto Nacional de Antropología e Historia dirigió varias investigaciones arqueológicas en sitios reportados desde los años cuarenta. El caso más importante es en 1975, con el inicio de los trabajos en Cacaxtla, lugar que por sus destacadas pinturas murales recibió una gran atención que se extendió hasta 1982.

Después de estos trabajos en 1992 se generó el llamado proyecto Xochitecatl, que a partir de la creación del Fondo Nacional Arqueológico y junto con otros doce proyectos más, revaluaron parte del patrimonio arqueológico de nuestro país. El proyecto Xochitecatl se dedicó a explicar la dinámica cultural del sitio con relación a su entorno natural y social, reconstruyendo los patrones de conducta cotidiana durante las diversas fases ocupacionales y conocer por qué se escogió ese lugar

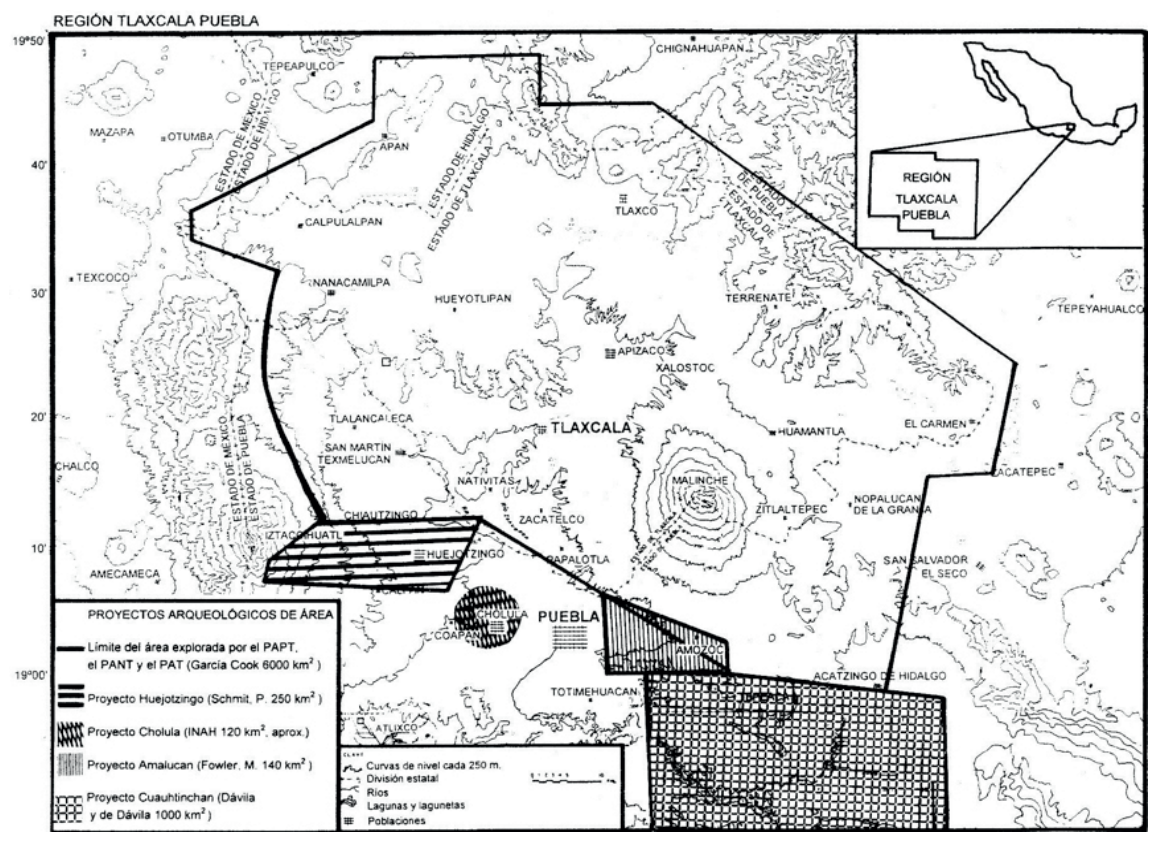

Fig. 5. Localización de las áreas de recorrido de los proyectos Puebla-Tlaxcala, Huejotzingo, Cholula y Amalucan de los años setenta. Tomado de García Cook:1997a, anexo cartográfico. 
para erigir Cacaxtla-Xochitecatl y su relación con los asentamientos del mismo periodo presentes en el valle Puebla-Tlaxcala (Serra et al 1998).

El proyecto Xochitecatl también emprendió nuevas excavaciones en el sitio de Cacaxtla (Plaza de los tres Cerritos, 1993-1994) y en 1996, deriva en un segundo proyecto: El hombre y sus recursos en el valle de Tlaxcala durante el Formativo y el Epiclásico Con este proyecto se realizó un nuevo recorrido, pero ahora solamente en el bloque geográfico Xochitecatl-Nopalucan-Nativitas cuyo propósito fue encontrar unidades habitacionales asociadas con el sitio principal de Xochitecatl-Cacaxtla. En las lomas del Municipio de Nativitas se hallaron los restos de unidades habitacionales que se han venido excavando de forma intensiva y extensiva desde 1998 hasta la fecha. Estos estudios han aportado información pertinente a la vida cotidiana productiva de los habitantes comunes de Xochitecatl-Cacaxtla durante el periodo Formativo o Preclásico (400 AD - 200 AD) y el Epiclásico (650-950 AD) (Serra y Lazcano 2011).

Condiciones sociales prehispánicas en el valle Puebla-Tlaxcala

La historia de las sociedades que habitaron lo que hoy conocemos como Mesoamerica, nos habla de una estabilidad y equilibrio que fueron interrumpidos por transformaciones y cambios sociales (como lo fue la transición de las sociedades tribales a sociedades clasistas-estatales). El valle Puebla-Tlaxcala ha sido considerado un claro ejemplo de lo anterior, ya que durante el periodo Preclásico esta región fue uno de los primeros lugares en toda Mesoamérica donde se dio la explotación agrícola. Diversos sistemas y técnicas de cultivo se pusieron en práctica, lo que aunado a la fertilidad del área, permitió que el sustento humano estuviera garantizado. La actividad agrícola trajo cambios en la organización social y en las actividades antes realizadas, con lo cual se desarrollaron nuevas formas de visualizar y de entender el mundo. Durante este periodo los asentamientos surgieron por toda el área y la concentración de unidades de residencia generó pequeñas aldeas y villas, algunas de las cuales destacaron sobre otras y se convirtieron en los si- tios principales de la región como: Tlalancaleca, Xochitécatl, Cholula, Cantona entre otros.

A finales del periodo Preclásico $(200 \mathrm{dC})$ se dieron cambios sociales fundamentales, ya que en ese momento se identifica la presencia de sociedades con instituciones estatales, a diferencia de los grupos tribales que estuvieron presentes durante el Preclásico temprano. A pesar de que el sustento agrícola siguió siendo prioritario, para el Preclásico tardío las actividades se diversificaron ampliamente, y la interacción no fue solamente con grupos de la región, sino también con grupos del área de Oaxaca y del área Maya (Serra y Lazcano 2011: 45).

Otro proceso social se dio durante el llamado paso del Clásico al Epiclásico. Mientras el periodo Clásico fue un largo momento durante el cual las sociedades tuvieron estabilidad y un dominio macro regional (Teotihuacan), el Epíclásico representa un proceso transicional que se caracteriza por un ordenamiento más bien de tipo local, ejemplificado por sitios como Teotenango, Xico y Santa Cruz Atizapán en el Estado de México; Xochitécatl-Cacaxtla en Tlaxcala, Xochicalco en Morelos, Cantona en Puebla, Chapantongo en Hidalgo, Tula en sus primeros momentos y posiblemente Cholula. Asimismo, surgieron nuevas interpretaciones sobre los procesos que se dieron en esta región durante todo el periodo prehispánico, para lo cual se utilizaron como ejemplo los casos específicos de tres sitios arqueológicos que probablemente dominaron la región (Sanders 1989:211-215; Cohodas 1989:222-227; López y López 2001).

La primera interpretación se refiere al sitio arqueológico de Cholula, concebido como Tollan, fue uno de los principales centros urbanos del Altiplano, Este lugar tuvo la capacidad de congregar población, y al estar situado en un área de paso obligado (al sur del valle Puebla -Tlaxcala), llegó a convertirse en un gran centro ceremonial. Desde 200 aC continúa creciendo para posteriormente compararse con sitios como Teotihuacan, y se mantiene así durante el Epiclásico, para concluir su ocupación en el Postclásico tardío. De igual forma se considera como un centro que controlaba el intercambio interregional de materiales que entraban por la zona oriental, así como de las rutas que conectaban a este 
centro con las diversas áreas mesoamericanas (Mountjoy 1987; Uruñuela y Plunket 2005).

La segunda interpretación se basa en el tipo de interacción que se dio al interior de la región. En el extremo de la zona noroeste de la región Puebla-Tlaxcala se hizo presente una gran ciudad: Cantona, urbe compleja, la más grande en aquel momento, la cual, durante su apogeo tuvo alrededor de $13 \mathrm{~km} 2$ y contó con unos 80 mil habitantes (García Cook 1995). Con seguridad, Cantona tuvo todo el control del "comercio" del Altiplano Central con el centro del Golfo y, por lo tanto, jugó un papel muy importante en esta región, que en su momento de apogeo cubrió alrededor (García Cook 1995).

La tercera interpretación se sustenta a partir del patrón de asentamiento que se manifiesta desde el periodo Preclásico en el llamado Bloque Xochitécatl-Nativitas-Nopalucan, cuya disposición establece una clara jerarquía, espacio geográfico localizado en la región noroeste del valle y ocupado por el sitio Cacaxtla-Xochitécatl-Nativitas. Los sitios que rodean Cacaxtla-Xochitécatl se ubican en lugares elevados y en terrazas bajas en estrecha relación con estos sitios principales.

La información que aquí presentamos en forma resumida señala los temas que fueron abordados, algunos con poca profundidad y otros, que resultan fundamentales para lograr un cabal conocimiento del cambio y desarrollo social de la región, han sido poco estudiados (vida cotidiana, actividades productivas, recursos explotados, entre otros).

\section{Estudios regionales su importancia y trascen- dencia}

El inicio de los estudios regionales y su importancia se atribuye a los primeros trabajos realizados por Willey (1953) con su proyecto en el valle de Perú. Su objetivo fue explicar los procesos adaptativos de las poblaciones en la región mencionada mediante el estudio del patrón de asentamiento. El mismo Willey da una primera definición de patrón de asentamiento:

"La manera en que el hombre dispone de sí mismo en el paisaje o medio en que él habita. Se refiere a casa, a sus arreglos y a la naturaleza y disposición de otras estructuras pertenecientes a la vida comunitaria. Estos asentamientos reflejan el medio ambiente natural, el nivel de tecnología y varias instituciones de interacción social y control que la cultura mantiene. Como los patrones de asentamiento son, a grandes escalas, directamente configurados por las necesidades culturales, ellos pueden ofrecer un punto de partida estratégico para la interpretación funcional de culturas arqueológicas" (Willey 1953 apud Sugiura 2009:91).

Se considera que esta definición sigue vigente, a pesar de los matices que otros autores han propuesto al respecto. El estudio del patrón de asentamiento se sustenta implícita o explícitamente en el supuesto de que los elementos conformadores de una organización sociopolítica son reflejo de la forma particular en que los grupos humanos se establecen en un espacio determinado; es decir, si consideramos que los fenómenos arqueológicos se caracterizan por patrones distribucionales, y que éstos son consecuencia directa o indirecta de la conducta humana del pasado. De esta manera, es posible acercarse a un tipo específico de organización social mediante el estudio de patrones distribucionales de la cultura material (Sugiura 2005:28).

El resultado de este tipo de proyectos ha demostrado que una investigación a escala macro posee una enorme eficacia como herramienta metodológica en la arqueología. El estudio regional de patrón de asentamiento tiene limitaciones en sus planteamientos inherentes, pero se reconoce que la arqueología de superficie es un recurso insustituible como primera aproximación a la realidad arqueológica de nivel macro y, sin duda, cuenta con el potencial para ponderar implicaciones sociales, económicas y políticas, así como macroprocesos históricos de la sociedad bajo estudio (Sugiura 2009).

De esta manera, nuestra investigación se sustenta en un punto de vista regional, considerando que la información producida por los trabajos mencionados representa un primer acercamiento a la realidad social que nos interesa investigar. Por ello realizaremos un recorrido 
de superficie en toda el área de investigación, relocalizando los sitios arqueológicos reportados por el proyecto Puebla-Tlaxcala y registraremos aquellos que no fueron observados. Toda esta información permitirá elaborar una base de datos con las características observadas, acompañadas por mapas del patrón de asentamiento. Cabe reiterar que el proyecto Puebla-Tlaxcala, así como los proyectos Cuauhtinchan, Huejotzingo y Cholula que se realizaron en los años setenta no abarcaron la totalidad del área que cubre el valle Puebla Tlaxcala. Una franja al centro-este, así como una pequeña elevación al este de la actual ciudad de Puebla, quedaron sin investigación.

\section{Fundamentación teórica}

Con el objetivo de conocer los agentes sociales y de inferir las causas de los cambios en el área y momento histórico de estudio, utilizamos una categoría cuyo contenido incluye los elementos principales que toda la sociedad pretérita y presente tiene y que se refiere a su organización económico-social ${ }^{1}$. Ésta permite conocer las particularidades de una sociedad tomando en cuenta las condiciones técnicas de producción (ambiente, recursos, etc.) y las condiciones sociales de la producción (sistema político, sistema de parentesco, etc.). Es decir, la suma de acciones emprendidas y alcanzadas por un grupo social para satisfacer sus necesidades y que debemos inferir a partir de los contextos arqueológicos y evidencia empírica que ha llegado hasta nosotros. De esta manera consideramos necesario conocer las sociedades y grupos a través de su organización económica y social ${ }^{2}$, lo cual implica conocer las formas y los recursos naturales que se explotaron; inferir las particularidades y las similitudes de cada grupo; definir el uso del espacio a

1 Para tener una definición amplia de estas categorías consultar a Bate:1998.

2 La organización económica social da cuenta de los procesos económicos del modo de producción (producción, cambio, consumo, distribución) gran escala (área pública, área residencial, área ceremonial, área productiva y habitacional); identificar las actividades productivas principales (modos de trabajo); definir las áreas de sostenimiento agrícola y de aprovisionamiento de alimento; conocer el tamaño y la densidad de la población, su composición étnica, su especialización económica, los tipos de interacción, así como los símbolos y la cosmovisión representativos. Estos aspectos se estudiaran desde una perspectiva regional y se ubicarán en el periodo Epiclasico ya que para este momento se tiene la mayor información a partir de los trabajos realizados en el sitio de Xochitecatl-Cacaxtla-Nativitas, Cantona, Cholula y otros y con los cuales se realizarán comparaciones.

\section{Objetivo General}

De esta manera nuestro objetivo general es conocer la localización, jerarquía, condiciones de conservación y características culturales y económico-social de los asentamientos del periodo Epiclasico en el valle Puebla-Tlaxcala.

\section{Metodología y Técnicas}

El recorrido de superficie se adecuó según las características propias del valle Puebla-Tlaxcala: Limita al norte con las laderas escarpadas del bloque de Tlaxcala. Hacia el poniente se eleva suavemente hasta alcanzar las faldas de la Sierra Nevada; por el oriente termina donde comienzan las espaciosas faldas del volcán Malinche y, por el sur, se pierde en el estado de Puebla (véase fig. 6). La Malinche señala el límite entre las secciones poblana y tlaxcalteca del valle, éste se extiende por el poniente de esa montaña hacia el estado de Tlaxcala, donde es separado por pequeñas serranías de la región de los Llanos de Apan. Respecto de sus características geográficas, el valle presenta en promedio un clima templado y lluvias moderadas. Tiene una altitud media de 2160 metros sobre el nivel del mar. Pertenece a la provincia geológica del Eje Neovolcánico, y 
está caracterizado por la presencia de altas cumbres originadas entre los períodos terciario y cuaternario. Es muy extenso y hasta hace poco alojaba varios llanos menores. Su paisaje está lleno de campos fértiles, separados por hileras de árboles añejos que sombrean caminos y canales. En algunos lugares todavía quedan algunas ciénegas y lagunas, como es el caso de Acuitlapilco, rodeada por tulares. Casi no queda restos de su vegetación original, ya que fue sustituida por plantas útiles al hombre. Varias de sus lagunas fueron desecadas para dedicarlas al cultivo, pero los subsuelos grises y manchados por la acción del agua testimonian su pasado lacustre. Los dos ríos más importantes del Estado atraviesan el valle: el Atoyac y el Zahuapan, que se une más abajo al primero. El área de estudio cubre aproximadamente 1 $600 \mathrm{~km} 2$ de los cuales $700 \mathrm{~km} 2$ se encuentran cubiertos por poblaciones actuales como la ciudad de Puebla de Zaragoza y zona metropolitana (Gutiérrez et al: 2003) (véase fig. 7).

\section{La estrategia metodológica de investigación estableció el siguiente trabajo:}

\section{a. Reconocimiento sistemático de superficie.}

Este tipo de recorrido está enfocado a la detección total de elementos arquitectónicos y áreas de actividad humana. Asimismo se ubicaron los sitios ya reportados por las investigaciones de la FAIC (Fundación Alemana). Se verificó la existencia de sitios y se ubicaron con nuestro sistema de registro que consiste en sectores de $10 \times 10 \mathrm{~km}$. que se subdividieron en $500 \mathrm{~m}$ los cuales se recorrieron a una distancia máxima de $100 \mathrm{~m}$ (véase fig. 8).

El recorrido se efectuó en el cien por ciento del área aquí considerada (entre 50 o 60 por ciento del total del área del valle se encuentra ya urbanizado) delimitando y registrando los sitios no reportados. Se tomarán como indicadores la evidencia topográfica de más de $1.5 \mathrm{~m}$ del nivel promedio del área, presencia de materiales arqueológicos y aquellos utilizados para la construcción (estuco, piedra, etcétera). b) Recolección de materiales arqueológicos de superficie.

Con el fin de obtener una idea precisa sobre la distribución general y posible cronología del área, la recolección del material se realizó por sitio.

\section{c) Localización y registro de cada sitio.}

Esto se realizó con base en las cédulas establecidas por el Instituto Nacional de Antropología e Historia que contiene: nombre del sitio, localización georeferenciada, descripción del sitio, médio ambiente y datos generales como: dimensiones, cronología, tipo de material, uso actual del suelo, estado de conservación, entre otros.

\section{Resultados}

Con base en estas actividades se alcanzaron los siguientes objetivos:Se estableció que la mayoría de los sitios arqueológicos localizados en el valle Puebla-Tlaxcala si tuvieron una ocupación del perido Epiclásico.

Se conoció las características culturales y estado de conservación de cada uno de los sitios.

Se reconstruyó y diferenció el patrón de asentamiento en el área de estudio.

Se estableció la relación que existió entre los habitantes de estos sitios epiclásicos y el medio que explotaron.

Se conoció la extensión y los límites del conjunto total de los sitios del periodo Epiclásico.

Se definió la jerarquía y la existencia de sitios rectores en el área del valle Puebla-Tlaxcala.

Se determino la presencia de por lo menos 116 sitios, de los cuales el denominado San Pablo del Monte es el indicado para realizar una futura exploración (figs 9 y 10).

Los sitios que aún perduran en el área de estudio se localizan en zonas agrícolas bajas, de pie de monte y pequeñas serranías, todos ellos cercanos a pequeños arroyos o fuentes de agua. Varios de ellos presentan pequeños montículos de dos a tres metros de altura que conforman pequeñas plazas con casas en su alrededor (fig. 11). Otros más, son grandes concentra- 
ciones de material arqueológico producto de la destrucción de los edificios principales. El patrón de asentamiento establece dos características principales. La primera de ellas se refiere a la nucleación de sitios menores en torno al centro rector de Xochitecatl-Cacaxtla, no así al sitio principal de Cholula. La segunda tiene que ver con un patrón de asentamiento en el que se prefieren corredores naturales de tránsito y de acceso, lejanos a zonas altas, lugar donde se establece la presencia de sitios para ritualidad y no habitacional.

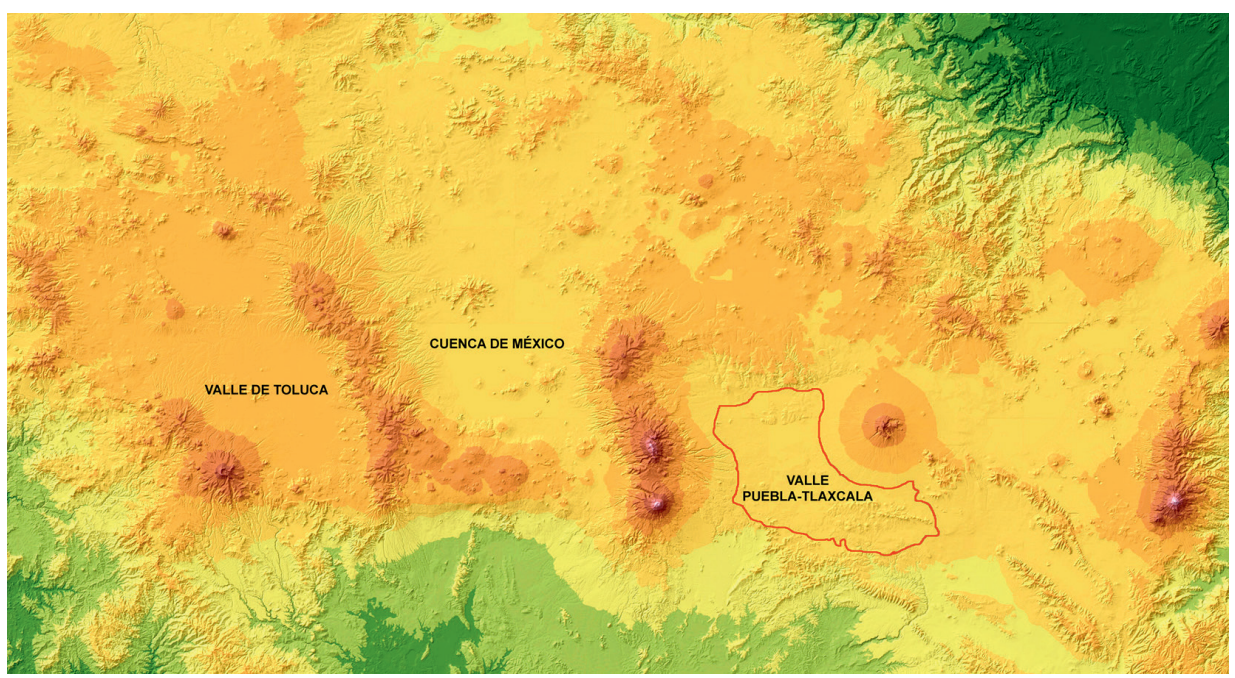

Fig. 6. Localización del valle Puebla-Tlaxcala con respecto a otras áreas geográficas del altiplano central mexicano.

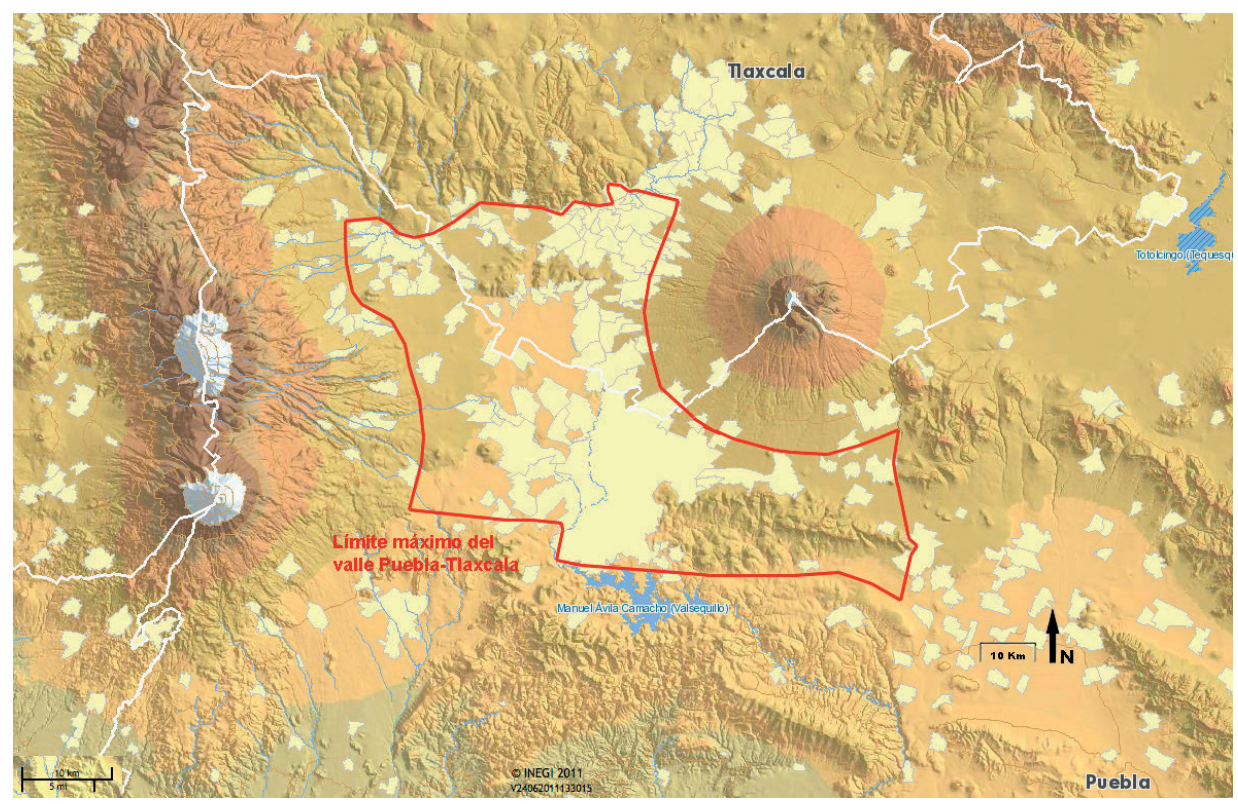

Fig. 7. Localización del valle Puebla-Tlaxcala y la zona urbana actual. 


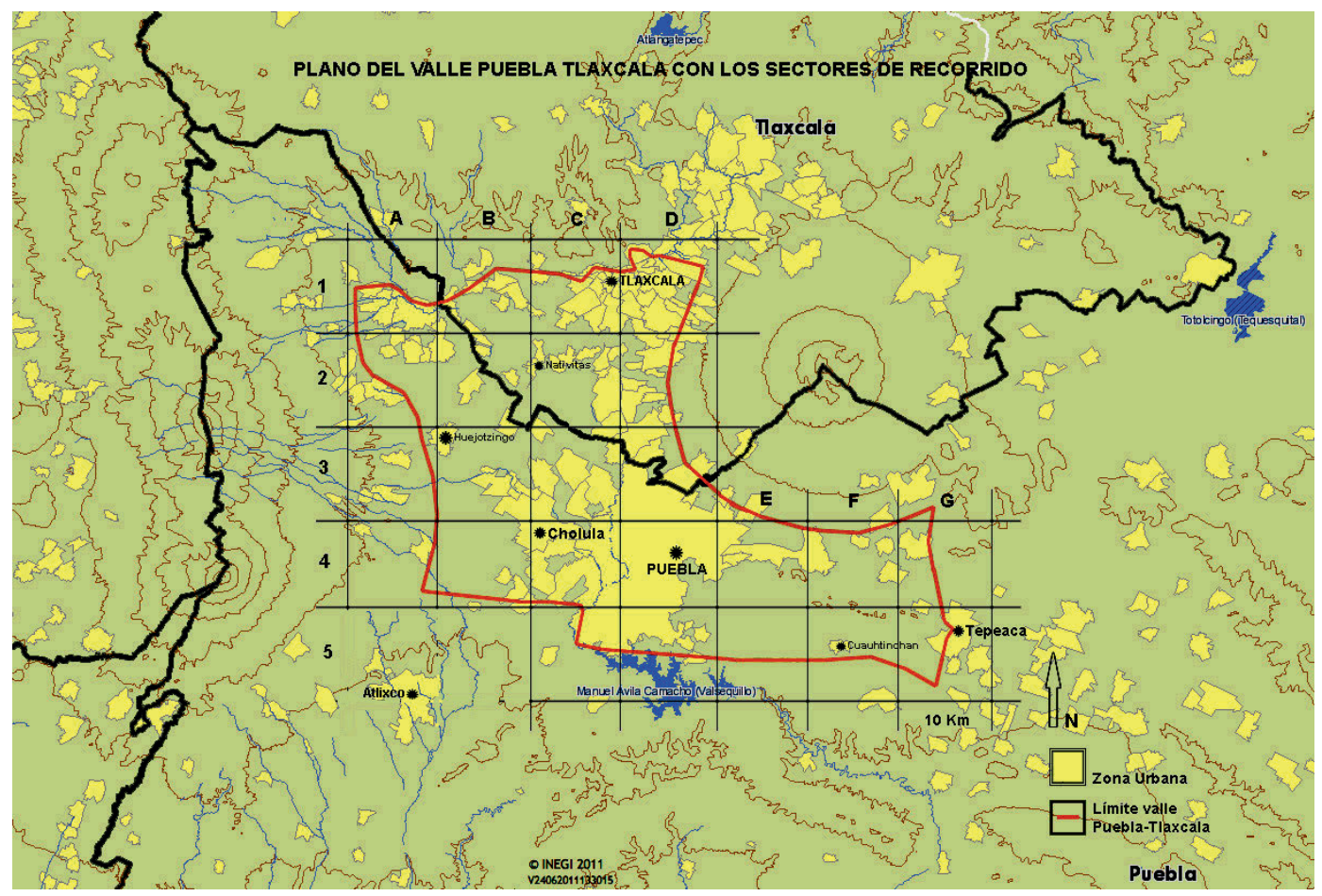

Fig. 8. División en sectores para el recorrido del área total del valle Puebla-Tlaxcala.

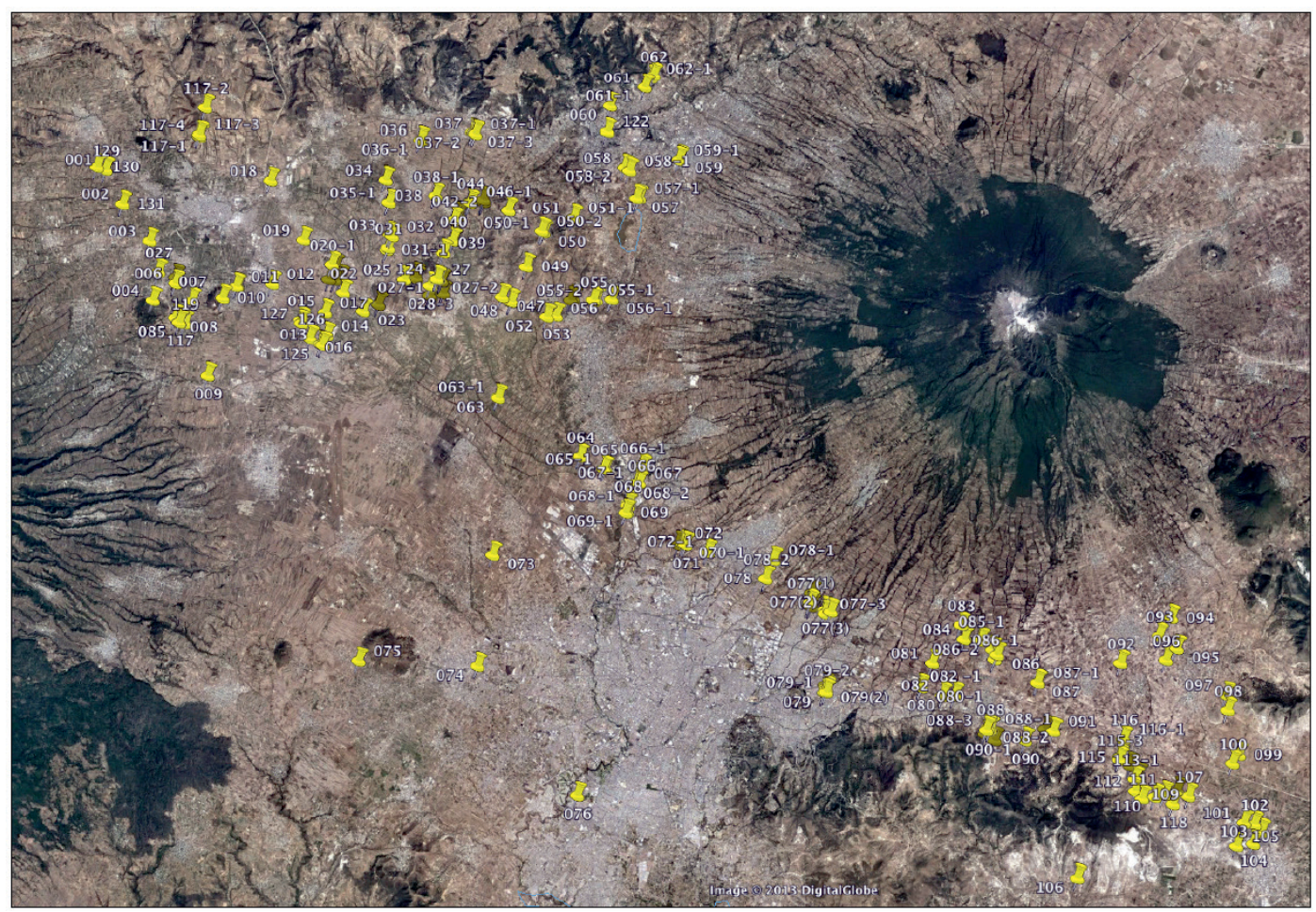

Fig. 9. Total de sitios localizados en el valle Puebla-Tlaxala y su ubicación respecto al área urbana actual. 


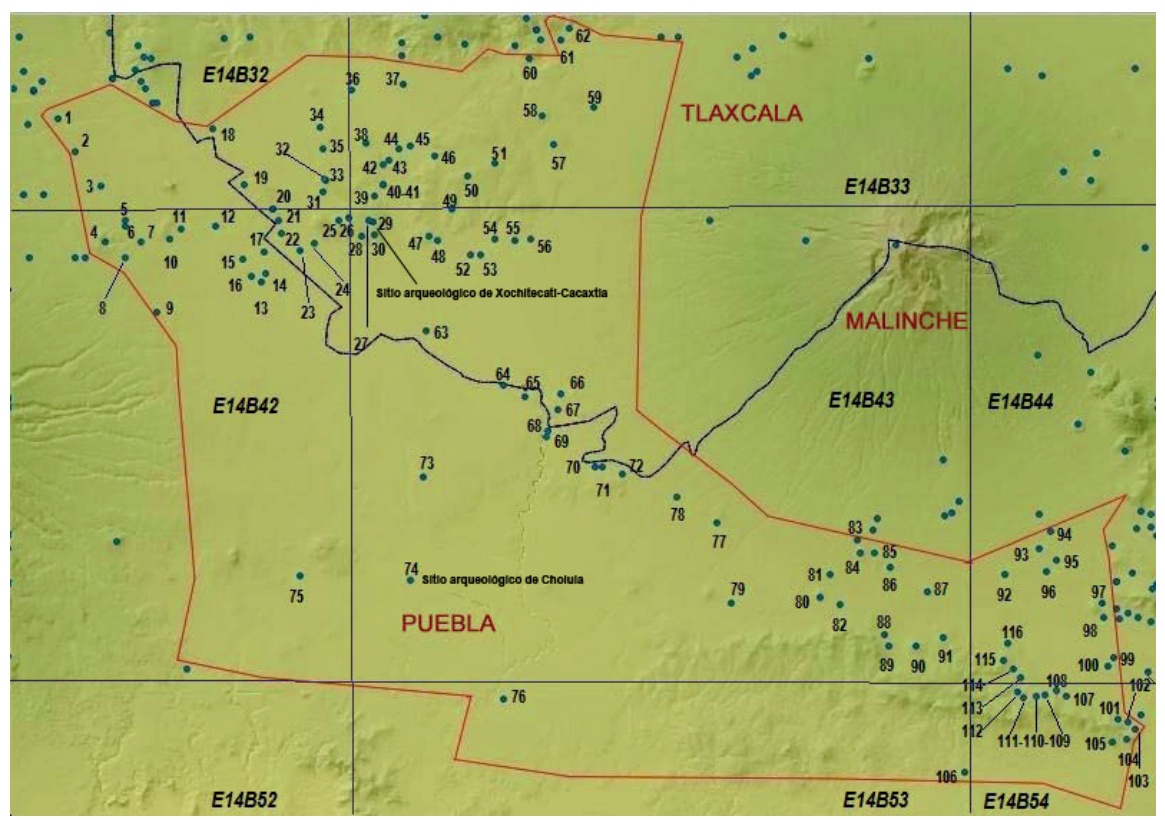

Fig. 10. Se constató la ubicación y condiciones de conservación de 116 sitios arqueológicos, varios de los cuales se encuentran en mal estado de conservación.
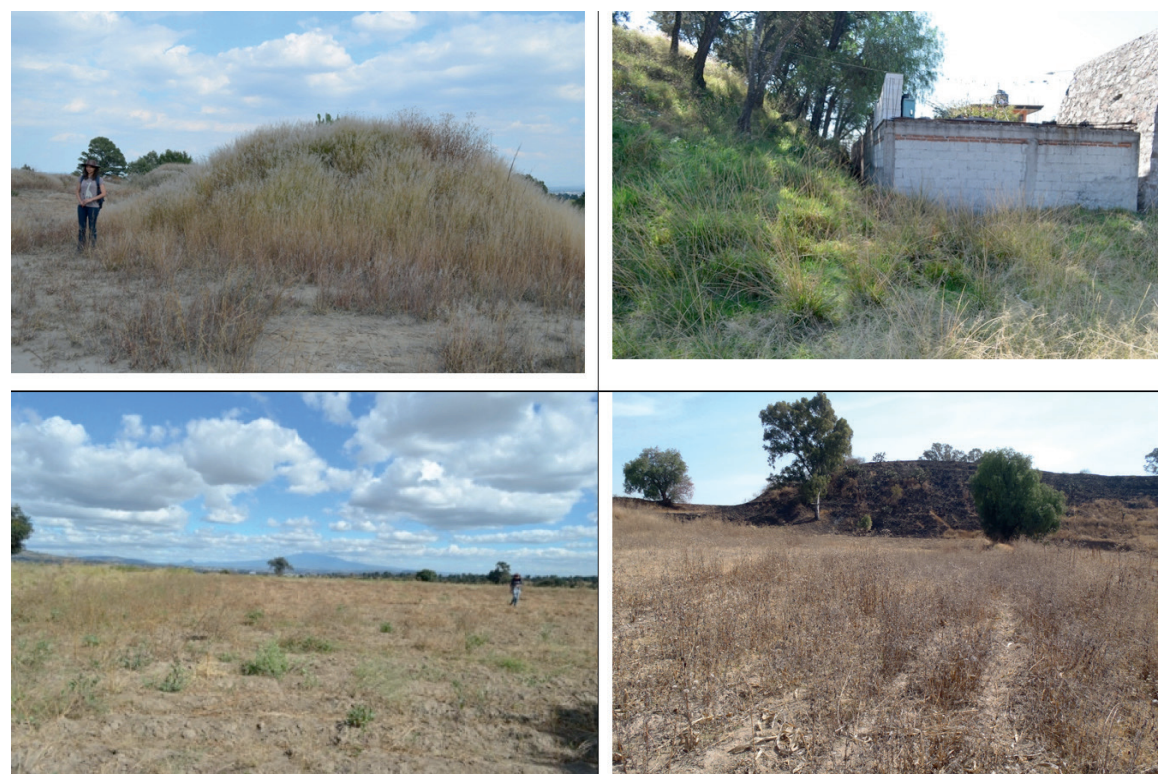

Fig. 11. A - Sitio llamado Tlamaoc constituido por varios montículo de 2 a 3 metros de altura. Su estado de conservación es malo; B - Sitio de Tenatitla, (número 60). Pequeño montículo de 6 metros de altura cuyos alrededores se encuentra afectado por construcciones modernas; C - Sitio denominado como Caula que presenta grandes concentraciones de material, pero no así estructuras o pequeños montículos; D - Sitio monumental de San Juan Mixco. Plataforma prehispánica de más de 10 metros de alto con un montículo central de 25 metros de diámetro. Sitio principal que se encuentra muy saqueado y cuya superficie se utiliza para el cultivo de maíz. 


\section{Agradecimientos}

Durante los trabajos de campo participó la alumna Indira Menchaca Vargas de la Escuela Nacional de Antropología e Historia a quien le agradecemos el apoyo brindado. Asimismo señalar que este proyecto de investigación fue realizado gracias al Programa UNAM-DGAPA -PAPIIT IN401814, México.

LAZCANO ARCE, J.C., SALlUM, M. Padrão de Assentamento dos Grupos que Habitaram o Vale de Puebla-Tlaxcala, México durante o Período Epiclássico (650-950 AD). Fase de Prospecção de superfície. R. Museu Arq. Etn., 25: 45-60, 2015

Resumo: $\mathrm{O}$ presente trabalho tem como objetivo conhecer, analisar e entender o padrão de assentamento dos sítios arqueológicos do período Epiclássico (650950 A.D.), localizados no vale de Puebla-Tlaxcala (México). A intensão primeira foi avaliar as condições de conservação em que se encontravam os sítios e, em segundo determinar quais as ligações de dependência segundo as características arquitetônicas e a área natural que estavam localizados, com o sítio de Xochitecalt-Cacaxtla. Para tanto foram utilizadas diversas fontes de informação: uma inicial, sustentados nos trabalhos prévios de recorrido de superfície e escavações realizadas em diversos sítios arqueológicos e uma segunda, sobre a base de novos dados coletados no atual estudo de prospecção de superfície. O estudo resultou no estabelecimento de uma área extensa de similitude cultural entre Xochitecatl-Cacaxtla e pelo menos vinte sítios na zona centro sul do vale cujo limite é a área nuclear do sítio principal de Cholula ao sul do próprio vale Puebla-Tlaxcala.

Palavras-chave: Vale de Puebla-Tlaxcala, Período Epiclássico, Mesoamérica

LAZCANO ARCE, J.C., SALLUM, M. Settlement Pattern of the Groups that Inhabites Valley Puebla-Tlaxcala, México during the Period Epiclasic (650-950 AD). Surface Exploration Phase. R. Museu Arq. Etn., 25: 45-60, 2015

Abstract: This paper aims to analyze and understand the settlement pattern of archaeological sites Epiclásico period (650-950 A.D.), located in the valley of Puebla-Tlaxcala (México). It is intended to assess first, the storage conditions in which they are and second, to determine which of them, according to the architectural characteristics presented and the natural area in which they are located, were dependent of the rector site Xochitecatl -Cacaxtla. Therefore, we used various sources of information: an initial, based on previous work surface and travels excavations at several archaeological sites and a second, on the basis of new data collected with the current study surface survey: To achieve this various information sources were used. The study succeeded in establishing a vast area of cultural similarity between Xochitecatl-Cacaxtla and at least twenty sites to the south-central valley region whose boundary is the area of the main nuclear site of Cholula south of Puebla-Tlaxcala valley itself.

Keywords: Puebla-Tlaxcala Valley, Epiclassic Period, Mesoamerica 


\section{Referencía Bibliografica}

Acuña, R. 1984 Relaciones Geográficas del Siglo XVI. Tlaxcala. Tomo I-II. México: UNAM.

Armillas, P. 1946. Los olmeca-xicalanca y los sitios arqueológicos del suroeste de Tlaxcala. Revista Mexicana de Estudios Antropológicos VIII: 137-145.

Bate, F.1998. El proceso de investigación en arqueología Barcelona: Crítica- Grijalbo Mondadori.

Clark, J.E. 1979. A Method for the Analysis of Mesoamerican Lithic Industries: An Application to the Obsidian Industry of La Libertad, Chiapas, Mexico. Tesis de Maestría. Salt Lake City: Brigham Young University.

Dávila Cabrera, J.M.P.1974. Cuauhtinchan: Estudio arqueológico de un área. Tesis Licenciatura. Ciudad de México: Escuela Nacional de Antropología e Historia, México.

Diehl, R.; Berlo, J.C. (Eds.). 1989. Mesoamerica after the decline of Teotihuacan A.D. 700-900. Washington DC:Dumbarton Oaks Resaarch Library and Colletion.

Dumon, D.E.; Müller, F. 1976. On Outline of the Demographic History of Tlaxcala. In Crawford, M.H. (ed). The tlaxcaltecans, Prehistory, demography, morphology and genetics. Publications in Antropology 7.Lawrence: University of Kansas.

Fowler, M.L. 1968. Un sistema Preclásico de distribución de agua en la zona arqueológica de Amalucan, Puebla. Puebla: Instituto poblano de antropología e historia.

García Cook, Á. 1972. Investigaciones arqueológicas en el Estado de Tlaxcala. Proyecto Puebla Tlaxcala, FAIC, Revista Comunicaciones 6.

García Cook, Á. 1976. El proyecto Puebla-Tlaxcala: finalidad y logros. Proyecto Puebla
Tlaxcala. Suplemento de Comunicaciones III. Puebla: FAIC.

García Cook, Á. 1995 Cruces de caminos. Desarrollo histórico de la región Poblano Tlaxcalteca. Revista de Arqueología Mexicana III (13):12-15.

García Cook, Á. 1997. El proyecto Puebla-Tlaxcala: orígen, finalidad y logros. In García Cook, A., Merino Carrión, L. (comps); Mirambell Silva, L. (coord). Antología de Tlaxcala. Colección Antologías, Serie Arqueología, Vol II,. INAH-Gobierno del Estado de Tlaxcala. p. 315-332.

García Cook, A. Merino, L. (comps.). 1991 Tlaxcala: textos de su historia. Los orígenes-arqueología. Vol. I y II. Beatriz Paredes (presentación). Gobierno del Estado de Tlaxcala.

García Cook, A. Merino, L. (comps.). 1996. Antología de Tlaxcala Antologías-Serie Arqueología Vol. I Lorena Mirambell (Coord) INAH, Gobierno del Estado de Tlaxcala.

García Cook, A. Merino, L. (comps.). 1997a Antología de Tlaxcala. Antologías-Serie Arqueología Vol. II, Lorena Mirambell (Coord) INAH, Gobierno del estado de Tlaxcala.

García Cook, A. Merino, L. (comps.). 1997b. Antología de Tlaxcala. Antologías-Serie Arqueología Vol. III, Lorena Mirambell (Coord) INAH, Gobierno del estado de Tlaxcala.

García Cook, A. Merino, L. (comps.). 1997c. Antología de Tlaxcala. Antologías-Serie Arqueología Vol. IV, Lorena Mirambell (Coord) INAH, Gobierno del estado de Tlaxcala.

García Cook, A. Merino, L. 1998. Cantona: Urbe Prehispánica en el altiplano central de México Latin American Antiquity 9(3):191-216. 
González-Jácome, A. 1993. Management of land, water and vegetation in traditional agro ecosystems in Central México. Landscape and Urban Planning 27:141-150.

Gutierrez Herreral L.; Cuervo Morales, M.J.; Ortiz Mendoza, E.O. 2003. Regiones naturales y de planeación para el estado de Puebla. Revista análisis económico XVIII (37):257-296.

Jimenez Moreno, W. 1942. El enigma de los Olmecas. Cuadernos Americanos V: 127-131.

Lazcano Arce, J.C.1998. Modos de Vida y explotación de recursos naturales en Cacaxtla Xochitécatl, durante el Epiclásico. Cuicuilco 5(14): 27-51.

Lazcano Arce, J.C. 2001. El pasado indigena. Fideicomiso Historia de las Américas, Serie Hacia una Nueva Historia de México. El Colegio de México, Fondo de Cultura Económica. México.

Lazcano Arce, J.C. 2005. Modo de vida y recursos naturales en el sitio de Nativitas, Tlaxcala durante el Epiclásico. Tesis de Maestría. Ciudad de Mexico: Escuela Nacional de Antropología e Historia.

Marquina, Ignacio (coord.).1970. Proyecto Cholula. Instituto Nacional de Antropología e Historia

Matos Moctezuma, E.; Pablo López,V. 1967. El edificio No.1 de Cholula. In Messmacher, M. (ed.) Cholula Reporte Preliminar. Ciudad de Mexico: Editorial. Nueva Antropología. p. 43-47.

Mountjoy, JB. 1987. La caída del Clásico en Cholula visto desde el Cerro Zapotecas. In Mountjoy, J.B.; Brockington, D.L. (eds.). El auge y la caída del Clásico en el México Central. Ciudad de Mexico: Instituto de investigaciones antropológicas, UNAM. p. 237-258.

Mountjoy, J.B.; Brockington, D.L. (eds.).1987 El auge y la caída del Clásico en el México Central.
Ciudad de Mexico: Instituto de investigaciones antropológicas, UNAM.

Plunket, P.; Uruñuela, G. 2006. Fechando Cholula. Informe FAMSI (não publicado). Arquivo digital disponível em http://www. famsi.org/reports/02042es/index.html.

Plunket, P. 1990. Arqueología y Etnohistoria en el valle de Atlixco. Notas Mesoamericanas.12: 3-18.

Schimdt, P.J. 1979. Investigaciones arqueológicas en la región de Huejotzingo-Puebla. Resumen de los trabajos del Proyecto Arqueológico Huejotzingo. In. Wilhelm, L.; Tyrakowski, K. (eds.). Procedimeintos del Segundo Simposio Proyecto Puebla-Tlaxcala. Comunicaciones 16:169-179.

Serra Puche, M. C. 1994 Xochitécatl In Proyectos Especiales de Arqueología, Consejo Nacional para la Cultura y las Artes, INAH, Fondo Nacional Arqueológico. p. 58-61.

Serra Puche, M.C.; Lazcano Arce, J.C. 1998 2010 Proyecto El hombre y sus recursos en el sur del valle de Tlaxcala durante el Formativo y el Epiclásico:. Sitio Nativitas. Informe Técnico de Excavación 1a, 2a $3^{\mathrm{a}}, 4^{\mathrm{a}}, 5^{\mathrm{a}}, 6^{\mathrm{a}}, 7^{\mathrm{a}}$, 8 , Temporadas (não publicado). Archivo Técnico del Consejo de Arqueología-INAH, México.

Serra Puche, M.C.; Lazcano Arce, J.C. 2005. El Epiclásico en el valle Puebla-Tlaxcala y los sitios de Cacaxtla-Xochitecatl-Nativitas . In Manzanilla, L. (ed.). Reacomodos demográficos del Clásico al Postclásico en el centro de México. Ciudad de Mexico: Instituto de Investigaciones Estéticas/Universidad Nacional Autónoma de México. p.287301

Serra Puche, M.C.; Lazcano Arce, J.C. 2011. Vida Cotidiana. Xochitecatl-Cacaxtla. Milenios. Años, Días (vol. I) Ciudad de Mexico: Instituto de Investigaciones Antropológicas-UNAM. 
Serra Puche, M.C.; Lazcano Arce, J.C.; de la Torre, M. 2009. Cerámica de Xochitecatl. Ciudad de Mexico: Instituto de Investigaciones Antropológicas-UNAM.

Solanes Carraro, M.C. 1995. Cholula. Revista de Arqueología Mexicana III (13): 24-30.

Snow, D.R. 1969. Ceramic Sequence and Settlement Location in Pre-Hispanic Tlaxcala. American Antiquity. 34:131-145.

Sugiura, Y. 2005. Y atrás quedó la ciudad de los dioses. Historia de los asentamientos del valle de Toluca. Ciudad de Mexico: IIA-UNAM.
Sugiura, Y. 2009. Caminando el valle de Toluca: arqueología regional, el legado de William T. Sanders. Revista Cuicuilco 47: 87-112.

Tschohl, P. 1977. Catálogo arqueológico y etnohistórico de Puebla-Tlaxcala, México. Tomo I. Freinburg-Köln.

Uruñuela, G.; Plunket, P. 2005. La transición del Clásico al Postclásico: Reflexiones sobre el valle Puebla-Tlaxcala. In Manzanilla, L. (ed.). Reacomodos demográficos del Clásico al Postclásico en el centro de México. Ciudad de Mexico: Instituto de Investigaciones Antropológicas-UNAM.p. 303-319. 